\title{
Ten-year survival and factors associated with increased mortality in patients admitted for acute decompensated heart failure in Thailand
}

\author{
Rungroj Krittayaphong ${ }^{1}$ MD, Prasart Laothavorn ${ }^{2}$, MD, Kriengkrai Hengrussamee ${ }^{3}$, MD, \\ Sopon Sanguanwong ${ }^{2}$, MD, Rapeephon Kunjara-Na-Ayudhya ${ }^{4}$, MD, Kasem Rattanasumawong ${ }^{5}, \mathrm{MD}$, \\ Chulaluk Komoltri ${ }^{6}$, PhD, Piyamitr Sritara ${ }^{7}, \mathrm{MD}$; Thai ADHERE Registry Group
}

INTRODUCTION Data on the long-term outcomes of Asian patients admitted for acute decompensated heart failure is scarce. The objectives of this study were to determine short-term, intermediate-term and long-term survival among patients admitted for acute decompensated heart failure in Thailand, and to identify factors independently associated with increased mortality.

METHODS Patients who were admitted with a primary diagnosis of heart failure were enrolled in the Thai Acute Decompensated Heart Failure Registry (ADHERE) from 18 hospitals located across Thailand during 2006. Medical record data was collected according to ADHERE protocol. Mortality data was collected from death certificates on file at the Thailand Bureau of Registration Administration.

RESULTS A total of 1,451 patients were included. The mean age of the patients was $63.7 \pm 14.4$ years, and $49.7 \%$ were male. One-year, five-year and ten-year mortality rates in Thai patients admitted for acute decompensated heart failure were $28.0 \%, 58.2 \%$ and $73.3 \%$, respectively. Independent predictors of increased mortality were identified. There were more cardiovascular-related deaths than non-cardiovascular-related deaths ( $54.6 \%$ vs. $45.4 \%$, respectively).

CONCLUSIONS The ten-year mortality rate in Thai patients admitted for acute decompensated heart failure was $73.3 \%$. Many factors were found to be independently associated with increased mortality, including left ventricular ejection fraction.

Keywords: acute decompensated heart failure, ADHERE registry, mortality, Thailand

\section{INTRODUCTION}

Heart failure $(\mathrm{HF})^{(1)}$ is a common condition with a prevalence of approximately $0.5 \%-2.0 \%$. Its prevalence has increased over the past decade, especially among the elderly. ${ }^{(4,5)} \mathrm{HF}$ accounts for $0.3 \%-3.0 \%$ of all primary diagnoses that require hospital admission. ${ }^{(3)}$ Despite advances in the management of $\mathrm{HF}$, the mortality rate remains high in both Western and Asian populations. ${ }^{(1,6)}$ HF-related mortality during hospital admission was reported in many studies to range from $3.8 \%$ to $9 \%$ in Western populations, and from $2 \%$ to $6 \%$ in Asian populations. ${ }^{(7}$ There are regional differences in HF outcome, which may be related to healthcare infrastructure, quality of care, access to healthcare and other environmental factors. ${ }^{(8)}$ Even within Asia, there are differences in the phenotypes of HF and comorbid conditions. $^{(9)}$ In Thailand, $6 \%$ of patients admitted with HF were reported to have died in hospital. ${ }^{(10)}$ It was estimated that $17 \%-45 \%$ of patients who were admitted with HF died within one year, and more than $50 \%$ died within five years, regardless of aetiology or gender. ${ }^{(3)}$

Data from the Asia-Pacific Acute Decompensated Heart Failure Registry (ADHERE) showed that the average age of patients admitted for HF in this region was approximately ten years less than that of patients in the United States and European
ADHERE. ${ }^{(11-13)}$ Additionally, their clinical presentation appeared to be more severe than in Caucasian populations. A better understanding of clinical presentation, survival/mortality, and comparisons with other countries and ethnicities is needed to improve the standard of care for HF patients in the Asia-Pacific region.

Accordingly, the aim of this study was to determine short-, intermediate- and long-term survival among patients admitted for acute decompensated HF in Thailand, and to identify factors independently associated with increased mortality in this patient population.

\section{METHODS}

The 2006 Thai ADHERE study included 18 medical centres from across Thailand and was conducted using a protocol similar to that in the ADHERE study conducted in the United States. Ten of the medical centres were university hospitals, three were government hospitals, and five were private hospitals. Patients aged $\geq 18$ years who were admitted with a primary diagnosis of HF were enrolled. Patients with cardiogenic shock, preoperative $\mathrm{HF}$ or HF as a secondary diagnosis, or comorbid conditions were excluded. The principal investigator at each centre was instructed to enrol consecutive patients. Index cases could be newly

\footnotetext{
Division of Cardiology, Department of Medicine, Faculty of Medicine Siriraj Hospital, Mahidol University, ${ }^{2}$ Division of Cardiology, Department of Medicine, Phramongkutklao College of Medicine, Bangkok, ${ }^{3}$ Department of Cardiology, Central Chest Institute of Thailand, Nonthaburi, ${ }^{4}$ Department of Cardiology, Vichaiyut Hospital and Medical Center, ${ }^{5}$ Police General Hospital, ${ }^{6}$ Division of Clinical Epidemiology, Department of Research and Development, Faculty of Medicine Siriraj Hospital, ${ }^{7}$ Division of Cardiology, Department of Medicine, Faculty of Medicine Ramathibodi Hospital, Mahidol University, Bangkok, Thailand

Correspondence: Dr Rungroj Krittayaphong, Head, Division of Cardiology, Department of Medicine, Faculty of Medicine Siriraj Hospital, Mahidol University, 2 Wanglang Road, Bangkoknoi, Bangkok 10700, Thailand. rungroj.kri@mahidol.ac.th
} 
diagnosed acute HF or chronic HF with decompensation. The diagnosis of HF was verified by a cardiologist in each included case. This study was approved by the institutional review board of each participating hospital. All patients gave written informed consent prior to participation.

The data for this study was derived from the 2006 ADHERE Thailand, with the aim of evaluating short-term (one-year), intermediate-term (five-year) and long-term (ten-year) mortality/ survival. Medical record data was collected from first contact at the emergency department to the time of hospital discharge, transfer to another hospital or in-hospital death. The following data was collected and recorded: demographic data; medical history; causes and precipitating causes of HF; clinical presentation; investigations including left ventricular ejection fraction (LVEF); hospital course; medication (oral and intravenous) given prior to, during and at hospital discharge; procedures; and death. An electronic data capture system was used to collect data from the participating medical centres. Data from each site was entered into an electronic case record form in the Web-based system. All data was verified to ensure accuracy and completeness. Longitudinal unique identifier (LUID) software was used to anonymise included patients. Each patient's LUID was stored in the database and used to track patients' hospital re-admission and clinical outcomes. In-hospital death and cause of death were recorded from the hospital discharge summary. Out-ofhospital mortality data, including date and cause of death, were collected from death certificates on file at the Thailand Bureau of Registration Administration.

Demographic and clinical data were summarised using descriptive statistics. Continuous data was presented as mean \pm standard deviation, while categorical data was presented as number and percentage. Student's $t$-test was used to compare continuous unpaired data, and chi-square test was used to compare categorical data. Univariate and multivariate Cox proportional hazard ratio analysis was performed to identify factors independently associated with increased risk of mortality. Variables with a p-value $<0.2$ in univariate analysis were included in multivariate analysis. A p-value $<0.05$ in multivariate analysis was considered statistically significant. All data analyses were performed using IBM SPSS Statistics version 20.0 (IBM Corp, Armonk, NY, USA).

\section{RESULTS}

A total of 1,451 patients were included. Their mean age was 63.7 \pm 14.4 years, and $49.7 \%$ were male. Baseline characteristics were compared between patients who survived and those who died during the ten-year follow-up period (Table I). There were a total of 1,064 deaths at ten years, an overall mortality rate of $73.3 \%$. Many parameters from the medical history, physical examination and laboratory data subsections were found to be associated with increased risk of mortality. These included diabetes mellitus (survived $31.0 \%$ vs. deceased $50.3 \% ; p<0.001$ ), hypertension (survived $48.1 \%$, deceased $67.1 \% ; p<0.001$ ), rales (survived $79.1 \%$, deceased $85.2 \% ; p=0.006$ ), LVEF (survived $48.3 \%$, deceased $45.4 \% ; p=0.011$ ) and serum creatinine (survived
$1.47 \pm 1.54 \mathrm{mg} / \mathrm{dL}$, deceased $1.95 \pm 1.75 \mathrm{mg} / \mathrm{dL} ; \mathrm{p}<0.001)$. Certain medications were found to be associated with decreased risk of death, such as angiotensin-converting enzyme inhibitors (ACEIs) or angiotensin receptor blockers (ARBs) (survived 59.2\%, deceased $42.6 \% ; \mathrm{p}<0.001$ ) and beta blockers (survived 30.2\%, deceased $23.0 \% ; p=0.005)$.

In our registry, very few patients $(1.9 \%, \mathrm{n}=28)$ received cardiac implantable electronic devices (CIEDs). Among them, three had a cardiac resynchronisation therapy defibrillator, three had an implantable cardioverter defibrillator, seven had a cardiac resynchronisation therapy device, and 15 had pacemakers.

The causes of death are shown in Table II. The most common cause of cardiovascular death was $\mathrm{HF}$, followed by myocardial infarction and stroke $(28.9 \%, 14.5 \%$ and $5.0 \%$, respectively, of those who died). The most common causes of non-cardiovascular death were sepsis, senility, renal failure and pneumonia $(14.3 \%$, $8.4 \%, 6.2 \%$ and $5.1 \%$, respectively, of those who died). LVEF data was available in 1,313 (90.5\%) patients. Of those, 505 (38.5\%) patients had LVEF $<40 \%$ (HF with reduced ejection fraction [HFrEF] group), and 808 (61.5\%) had LVEF $\geq 40 \%$ (HF with preserved ejection fraction [HFpEF] group).

Fig. 1 shows a forest plot for the unadjusted hazard ratios of factors that might be associated with an increase in ten-year mortality. Multivariate analysis was performed for factors that predicted ten-year mortality based on three levels of information: (a) Model 1, clinical information only; (b) Model 2, clinical information and investigation data; and (c) Model 3, clinical information, investigation data and medication data (Table III). When all three types of data were analysed together, elderly status, history of HF, stroke/transient ischaemic attack, use of cardiac devices, oedema, reduced ejection fraction and anaemia (haemoglobin $<12 \mathrm{~g} / \mathrm{dL}$ ) were found to be independent factors associated with increased risk of death. In contrast, taking either ACEls or ARBs was found to be an independent predictor of decreased mortality.

Survival graphs of patients in the Thai ADHERE relative to total death, cardiovascular death and non-cardiovascular death are shown in Fig. 2. More patients died of cardiovascular-related causes than non-cardiovascular-related causes (581 vs. 483, respectively) (Table IV). Overall mortality at one month, six months, one year, five years and ten years was $8.1 \%, 21.1 \%$, $28.0 \%, 58.2 \%$ and $73.3 \%$, respectively (Table IV). Survival curves comparing patients with HFrEF and those with HFpEF are shown in Fig. 3. Patients with HFrEF had higher mortality than patients with HFpEF, and the increased risk of death in patients with HFrEF was mainly related to cardiovascular causes.

\section{DISCUSSION}

The HF mortality rate was high in our study population at $8.1 \%$, $21.1 \%, 28.0 \%, 58.2 \%$ and $73.3 \%$, respectively, at one month, six months, one year, five years and ten years. Although many medications and CIEDs can significantly reduce mortality in patients with HF, mortality and re-admission rates remain high. ${ }^{(2,14)}$ In the Asia-Pacific region, patients with HF are reported to receive standard pharmacological treatment and device therapy at a suboptimal rate compared to patients in the West. ${ }^{(6,15)}$ 
Table I. Comparison of the baseline characteristics of patients who survived and those who died during the ten-year follow-up period.

\begin{tabular}{|c|c|c|c|c|}
\hline \multirow[t]{2}{*}{ Variable } & \multicolumn{3}{|c|}{ No. $(\%) /$ mean \pm standard deviation } & \multirow[t]{2}{*}{ p-value } \\
\hline & All patients $(n=1,451)$ & Survived $(n=387)$ & Deceased $(n=1,064)$ & \\
\hline Age (yr) & $63.7 \pm 14.4$ & $57.3 \pm 14.4$ & $66.0 \pm 13.6$ & $<0.001$ \\
\hline Male gender & $721(49.7)$ & $195(50.4)$ & $526(49.4)$ & 0.749 \\
\hline Body weight (kg) & $60.4 \pm 14.1$ & $63.1 \pm 15.5$ & $59.2 \pm 13.6$ & 0.001 \\
\hline \multicolumn{5}{|l|}{ Medical history } \\
\hline History of heart failure & $893(61.5)$ & $189(48.8)$ & $704(66.2)$ & $<0.001$ \\
\hline CAD & $606(41.8)$ & $116(30.0)$ & $490(46.1)$ & $<0.001$ \\
\hline History of MI & $345(23.8)$ & $62(16.0)$ & $283(26.6)$ & $<0.001$ \\
\hline Hypertension & $900(62.0)$ & $186(48.1)$ & $714(67.1)$ & $<0.001$ \\
\hline Diabetes mellitus & $655(45.1)$ & $120(31.0)$ & $535(50.3)$ & $<0.001$ \\
\hline Dyslipidaemia & $667(46.0)$ & $138(35.7)$ & $529(49.7)$ & $<0.001$ \\
\hline Stroke/TIA & $156(10.8)$ & $21(5.4)$ & $135(12.7)$ & $<0.001$ \\
\hline Pacemaker/ICD & $28(1.9)$ & $1(0.3)$ & $27(2.5)$ & 0.004 \\
\hline PAD & $43(3.0)$ & $4(1.0)$ & $39(3.7)$ & 0.008 \\
\hline CKD & $246(17.0)$ & $20(5.2)$ & $226(21.2)$ & $<0.001$ \\
\hline COPD/asthma & $100(6.9)$ & $16(4.1)$ & $84(7.9)$ & 0.012 \\
\hline Smoking & $98(6.8)$ & $32(8.3)$ & $66(6.2)$ & 0.166 \\
\hline \multicolumn{5}{|l|}{ Presentation/evaluation } \\
\hline NYHA Class IV & $929(64.0)$ & $230(59.4)$ & $699(65.7)$ & 0.028 \\
\hline Rales & $1,212(83.5)$ & $306(79.1)$ & $906(85.2)$ & 0.006 \\
\hline Oedema & $863(59.5)$ & $211(54.5)$ & $652(61.3)$ & 0.020 \\
\hline $\mathrm{SBP}(\mathrm{mmHg})$ & $136.3 \pm 32.1$ & $136.7 \pm 32.0$ & $136.1 \pm 32.1$ & 0.747 \\
\hline $\mathrm{DBP}(\mathrm{mmHg})$ & $78.6 \pm 20.2$ & $80.0 \pm 22.6$ & $78.1 \pm 19.3$ & 0.142 \\
\hline Heart rate (beats/min) & $93.6 \pm 22.9$ & $97.2 \pm 22.9$ & $92.3 \pm 22.8$ & $<0.001$ \\
\hline Pulmonary congestion & $1,283(88.4)$ & $341(88.1)$ & $942(88.5)$ & 0.825 \\
\hline LVEF (\%) & $46.2 \pm 18.2$ & $48.3 \pm 17.9$ & $45.4 \pm 18.3$ & 0.011 \\
\hline AF on ECG & $333(22.9)$ & $88(22.7)$ & $245(23.0)$ & 0.908 \\
\hline Q wave on ECG & $302(20.8)$ & $67(17.3)$ & $235(22.1)$ & 0.048 \\
\hline QRS width & $101.4 \pm 24.6$ & $95.5 \pm 16.9$ & $103.4 \pm 26.4$ & $<0.001$ \\
\hline Serum sodium (mEq/L) & $136.9 \pm 5.6$ & $137.5 \pm 5.2$ & $136.7 \pm 5.7$ & 0.014 \\
\hline BUN (mg/dL) & $28.2 \pm 20.5$ & $22.3 \pm 14.8$ & $30.2 \pm 21.9$ & $<0.001$ \\
\hline Creatinine (mg/dL) & $1.82 \pm 1.71$ & $1.47 \pm 1.54$ & $1.95 \pm 1.75$ & $<0.001$ \\
\hline Haemoglobin (g/dL) & $11.8 \pm 2.4$ & $12.4 \pm 2.1$ & $11.6 \pm 2.4$ & $<0.001$ \\
\hline Serum albumin (mg/dL) & $3.63 \pm 1.38$ & $3.78 \pm 1.46$ & $3.57 \pm 1.35$ & 0.029 \\
\hline \multicolumn{5}{|l|}{ Medication } \\
\hline Loop diuretic & $1,026(70.7)$ & $276(71.3)$ & $750(70.5)$ & 0.759 \\
\hline MRA & $216(14.9)$ & $57(14.7)$ & $159(14.9)$ & 0.919 \\
\hline ACEI/ARB & $682(47.0)$ & $229(59.2)$ & $453(42.6)$ & $<0.001$ \\
\hline Beta blocker & $362(24.9)$ & $117(30.2)$ & $245(23.0)$ & 0.005 \\
\hline Nitrate & $631(43.5)$ & $146(37.7)$ & $485(45.6)$ & 0.008 \\
\hline Digoxin & $364(25.1)$ & $106(27.4)$ & $258(24.2)$ & 0.222 \\
\hline Warfarin & $268(18.5)$ & $90(23.3)$ & $178(16.7)$ & 0.005 \\
\hline Antiplatelet & $840(57.9)$ & $208(53.7)$ & $632(59.4)$ & 0.054 \\
\hline Lipid lowering & $764(52.7)$ & $199(51.4)$ & $565(53.1)$ & 0.571 \\
\hline
\end{tabular}

ACEI/ARB: angiotensin-converting enzyme inhibitor/angiotensin receptor blocker; AF: atrial fibrillation; BUN: blood urea nitrogen; CAD: coronary artery disease; CKD: chronic kidney disease; COPD: chronic obstructive pulmonary disease; DBP: diastolic blood pressure; ECG: electrocardiogram; ICD: implantable cardioverter defibrillator; LVEF: left ventricular ejection fraction; MI: myocardial infarction; MRA: mineralocorticoid receptor antagonist; NYHA: New York Heart Association; PAD: peripheral arterial disease; SBP: systolic blood pressure; TIA: transient ischaemic attack

HF registry data has been reported from many countries, including the United States, Europe and Asia, such as Japan, Korea and Taiwan. ADHERE is a worldwide registry of patients admitted due to HF. Data from the Asia-Pacific region was earlier reported. ${ }^{(11)}$ Our data focused on patients enrolled in the ADHERE Thailand registry ten years ago, aiming to report long- 
Table II. Causes of death in the Thai Acute Decompensated Heart Failure National Registry $(n=1,451)$.

\begin{tabular}{|llll|}
\hline Cause of death & No. & \% of total & \% of deaths \\
\hline CV death & 581 & 40.0 & 54.6 \\
\hline Heart failure & 307 & 21.2 & 28.9 \\
\hline Myocardial infarction & 154 & 10.6 & 14.5 \\
\hline Valvular heart disease & 38 & 2.6 & 3.6 \\
\hline Arrhythmia & 23 & 1.6 & 2.2 \\
\hline Stroke & 53 & 3.7 & 5.0 \\
\hline Pulmonary embolism & 3 & 0.2 & 0.3 \\
\hline Systemic embolism & 3 & 0.2 & 0.3 \\
\hline Non-CV death & 483 & 33.3 & 45.4 \\
\hline Sepsis & 152 & 10.5 & 14.3 \\
\hline Pneumonia & 54 & 3.7 & 5.1 \\
\hline Renal failure & 66 & 4.5 & 6.2 \\
\hline Cancer & 41 & 2.8 & 3.9 \\
\hline Respiratory failure & 22 & 1.5 & 2.1 \\
\hline Bleeding & 9 & 0.6 & 0.8 \\
\hline Accident & 7 & 0.5 & 0.7 \\
\hline Diabetic complication & 7 & 0.5 & 0.7 \\
\hline Cirrhosis & 7 & 0.5 & 0.7 \\
\hline COPD/asthma & 8 & 0.6 & 0.8 \\
\hline Senility/unknown & 89 & 6.1 & 1.6 \\
\hline Others & 17 & 1.2 & 0.4 \\
\hline
\end{tabular}

COPD: chronic obstructive pulmonary disease; CV: cardiovascular

term mortality in this group. Despite the trend towards decreased mortality due to $\mathrm{HF}^{(16)}$ in-hospital mortality is still approximately $2 \%-7 \%$ in both Asian and Western populations. ${ }^{(6,17)}$ Specific to ADHERE, in-hospital mortality was $4 \%$ for the global ADHERE, ${ }^{(12}$ $4.8 \%$ for the Asia-Pacific ADHERE(11) and 5.5\% for the Thai ADHERE. ${ }^{(7)}$ The age at presentation of HF was found to be approximately eight years younger in the Asia-Pacific region than in other regions. In addition, disease severity was greater and mortality was higher in the Asia-Pacific ADHERE than in other ADHEREs. ${ }^{(11)}$ In the Thai ADHERE, in-hospital mortality was high compared to that of Western countries and other Asia-Pacific countries, a finding that may be partly related to disease severity and suboptimal treatment with standard medications. ${ }^{(6,7,11,12)}$

Poor outcomes were reported in both Western and Asian populations for patients with a diagnosis of HF. In the United States, HF mortality data at 30 days, one year and five years from the Atherosclerosis Risk in Communities study was 10\%, $22 \%$ and $42 \%$, respectively, ${ }^{(18)}$ which is lower than our rates at the corresponding time points. However, data from their Medicare patients revealed a one-year mortality rate of $32.2 \%$, slightly higher than our rate. A study from Australia reported $28 \%$ mortality at the one-year time point, which is similar to our finding. ${ }^{(19)}$ Although national and regional mortality rates cannot be directly compared due to differences in study population and local management practice, they reflect the overall malignant nature of the condition or combination of conditions referred to as HF. Another study showed that half of HF patients were re-admitted to the hospital within six months. ${ }^{(20)}$ Generally, few

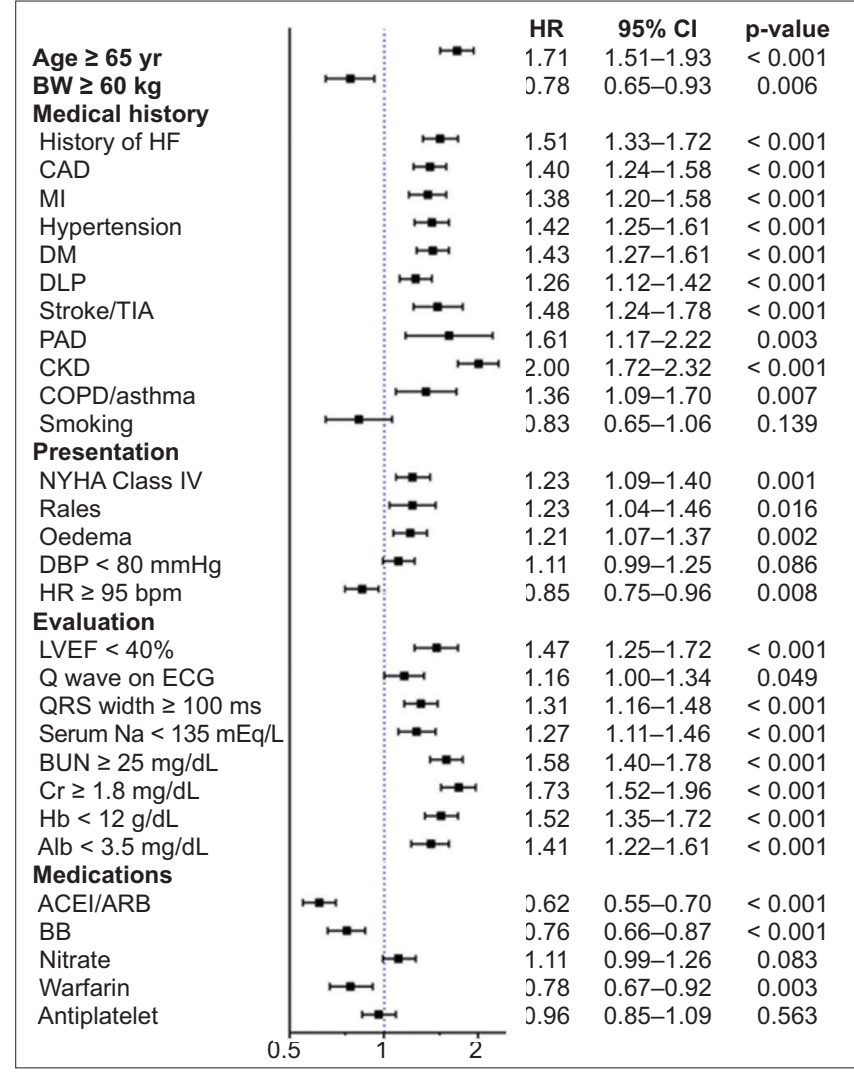

Fig. 1 Forest plot shows unadjusted hazard ratios of factors predicting mortality. ACEI/ARB: angiotensin-converting enzyme inhibitor/angiotensin receptor blocker; Alb: albumin; BB: beta blocker; BUN: blood urea nitrogen; BW: body weight; CAD: coronary artery disease; $\mathrm{Cl}$ : confidence interval; CKD: chronic kidney disease; COPD: chronic obstructive pulmonary disease; Cr: creatinine; DBP: diastolic blood pressure; DLP: dyslipidaemia; DM: diabetes mellitus; ECG: electrocardiogram; Hb: haemoglobin; HF: heart failure; HR: hazard ratio; LVEF: left ventricular ejection fraction; MI: myocardial infarction; Na: sodium; NYHA: New York Heart Association; PAD: peripheral arterial disease; TIA: transient ischaemic attack

studies have reported five- and ten-year mortality in HF. The fiveyear mortality rate of $58.2 \%$ in the present study is comparable to rates previously reported in the United States ${ }^{(16,21)}$ and the United Kingdom. ${ }^{(22,23)}$ Our ten-year mortality rate of $73.3 \%$ is comparable to a rate reported in Scotland. ${ }^{(23}$

Factors predicting long-term mortality in our study included older age, history of HF, stroke, use of cardiac electronic devices, oedema, low LVEF, anaemia and not receiving ACEIs/ARBs. Previous data also showed that elderly status and comorbidities, such as diabetes mellitus, renal dysfunction, anaemia and atrial fibrillation, were factors that predicted mortality and adverse outcomes in both Asian and Western populations. ${ }^{(24)}$ Low blood pressure itself does not contribute to mortality in $\mathrm{HF}$, but it is a surrogate marker for lower cardiac output state and intolerance to medications. ${ }^{(10,25)}$ Adherence to guideline-recommended medications has been shown to be associated with better outcomes. $^{(26)}$ Data from the BIOSTAT-CHF (BIOlogy Study to TAilored Treatment in Chronic Heart Failure) study demonstrated that ACEIs/ARBs and beta-blockers were positively associated with clinical outcome. ${ }^{(27)}$

Studies showed that Asian HF patients received standard medication at a much lower proportion than Western populations. ${ }^{(7,11,24,25)}$ Combined with the more severe clinical 
Table III. Multivariate analysis for independent factors predicting mortality based on three models* with different levels of information.

\begin{tabular}{|c|c|c|c|c|c|c|}
\hline \multirow[t]{2}{*}{ Variable } & \multicolumn{2}{|c|}{ Model 1} & \multicolumn{2}{|c|}{ Model 2} & \multicolumn{2}{|c|}{ Model 3} \\
\hline & HR (95\% CI) & p-value & HR (95\% CI) & p-value & HR (95\% CI) & p-value \\
\hline Age $\geq 65 \mathrm{yr}$ & $1.58(1.27-1.97)$ & $<0.001$ & $1.74(1.35-2.24)$ & $<0.001$ & $1.73(1.34-2.22)$ & $<0.001$ \\
\hline History of heart failure & $1.69(1.39-2.05)$ & $<0.001$ & $1.61(1.28-2.04)$ & $<0.001$ & $1.57(1.24-1.99)$ & $<0.001$ \\
\hline Diabetes mellitus & $1.23(1.01-1.49)$ & 0.039 & & & & \\
\hline Pacemaker/ICD & $2.12(1.27-3.55)$ & 0.004 & & & $2.12(1.14-3.94)$ & 0.017 \\
\hline PAD & $1.57(1.06-2.34)$ & 0.025 & & & & \\
\hline CKD & $1.72(1.35-2.19)$ & $<0.001$ & $1.44(1.07-1.94)$ & 0.017 & & \\
\hline Stroke/TIA & & & $1.45(1.02-2.06)$ & 0.038 & $1.47(1.03-2.09)$ & 0.032 \\
\hline Oedema & & & $1.35(1.07-1.70)$ & 0.012 & $1.40(1.12-1.76)$ & 0.004 \\
\hline LVEF $<40 \%$ & & & $1.33(1.06-1.66)$ & 0.013 & $1.47(1.17-1.84)$ & 0.001 \\
\hline Haemoglobin $<12 \mathrm{~g} / \mathrm{dL}$ & & & $1.39(1.09-1.75)$ & 0.007 & $1.44(1.15-1.79)$ & 0.001 \\
\hline ACEI/ARB & & & & & $0.63(0.50-0.79)$ & $<0.001$ \\
\hline
\end{tabular}

*Model 1: demographics and history, Model 2: Model 1 + physical exam and investigation, Model 3: Model $2+$ medication. ACEI/ARB: angiotensin-converting enzyme inhibitor/angiotensin receptor blocker; Cl: confidence interval; CKD: chronic kidney disease; HR: hazard ratio; ICD: implantable cardioverter defibrillator; LVEF: left ventricular ejection fraction; PAD: peripheral arterial disease; TIA: transient ischaemic attack
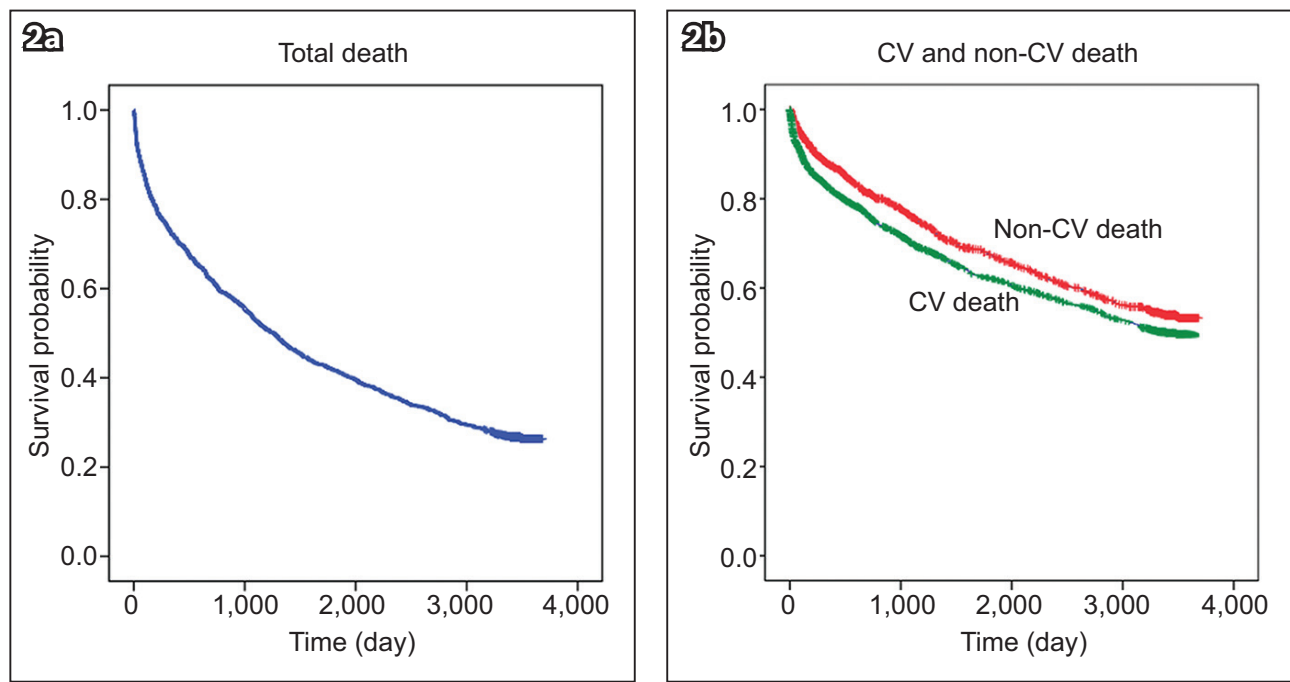

Fig. 2 Survival graphs show (a) all mortality; and (b) CV and non-CV mortality rates over time of patients in the Thai Acute Decompensated Heart Failure Registry. CV: cardiovascular
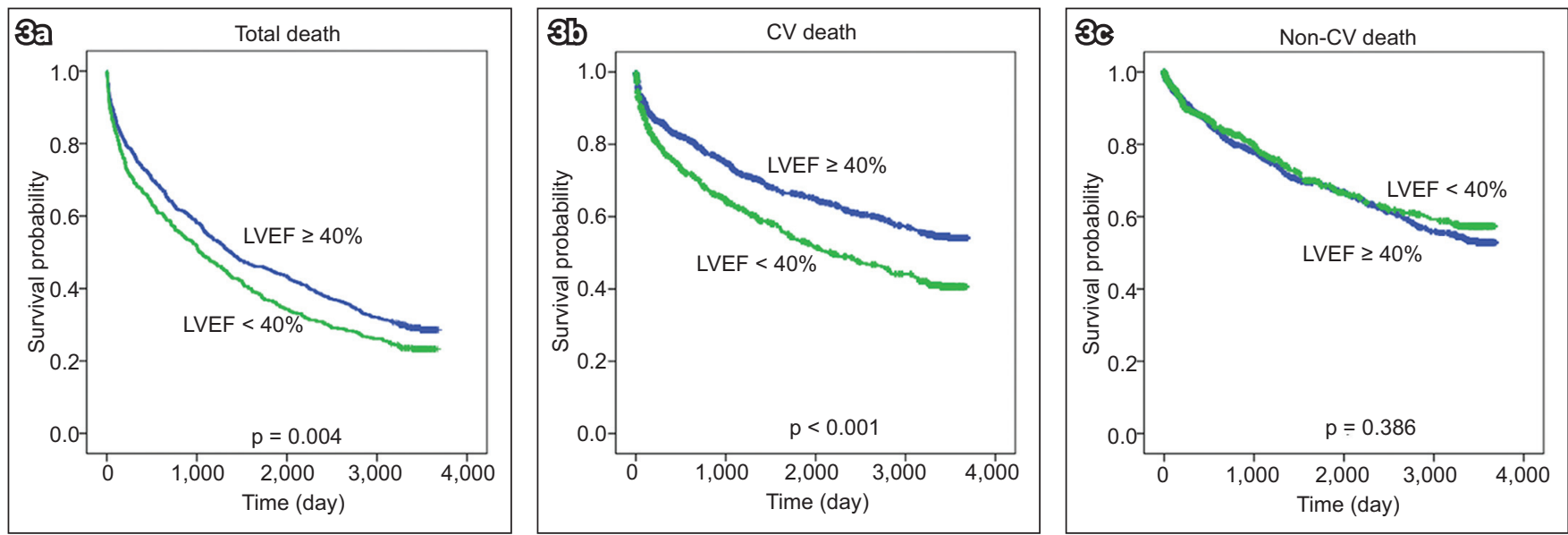

Fig. 3 Survival graphs show (a) all mortality; (b) CV; and (c) non-CV mortality rates over time of patients with low LVEF ( $<40 \%$ ) and preserved LVEF ( $\geq 40 \%$ ). CV: cardiovascular; LVEF: left ventricular ejection fraction 
Table IV. Total, CV-related and non-CV-related mortality compared between HFrEF and HFpEF mortality at various time points.

\begin{tabular}{|c|c|c|c|c|c|}
\hline \multirow[t]{2}{*}{ Type } & \multicolumn{5}{|c|}{ No. (\%) } \\
\hline & $1 \mathrm{mth}$ & $6 \mathrm{mth}$ & $1 \mathrm{yr}$ & $5 \mathrm{yr}$ & $10 \mathrm{yr}$ \\
\hline \multicolumn{6}{|l|}{ Total mortality } \\
\hline All $(n=1,451)$ & $118(8.1)$ & $306(21.1)$ & $407(28.0)$ & 845 (58.2) & $1,064(73.3)$ \\
\hline $\operatorname{HFrEF}(n=505)$ & $46(9.1)$ & $121(24.0)$ & 161 (31.9) & $320(63.4)$ & 387 (76.6) \\
\hline HFpEF $(n=808)$ & $57(7.1)$ & $151(18.7)$ & $205(25.4)$ & $442(54.7)$ & $574(71.0)$ \\
\hline \multicolumn{6}{|l|}{ CV death } \\
\hline All $(n=1,451)$ & $81(5.6)$ & $193(13.3)$ & $247(17.0)$ & $474(32.7)$ & $581(40.0)$ \\
\hline $\operatorname{HFrEF}(n=505)$ & $35(6.9)$ & $85(16.8)$ & $111(22.0)$ & 205 (40.6) & $245(48.5)$ \\
\hline HFpEF $(n=808)$ & $39(4.8)$ & 96 (11.9) & $122(15.1)$ & $235(29.1)$ & $293(36.3)$ \\
\hline \multicolumn{6}{|l|}{ Non-CV death } \\
\hline All $(n=1,451)$ & $37(2.5)$ & $113(7.8)$ & $160(11.0)$ & $371(25.6)$ & $483(33.3)$ \\
\hline $\operatorname{HFrEF}(n=505)$ & $11(2.2)$ & $36(7.1)$ & $50(9.9)$ & $115(22.8)$ & $142(28.1)$ \\
\hline HFpEF $(n=808)$ & $18(2.2)$ & $55(6.8)$ & $83(10.3)$ & $207(25.6)$ & $281(34.8)$ \\
\hline
\end{tabular}

$\mathrm{CV}$ : cardiovascular; $\mathrm{HFpEF}$ : heart failure with preserved ejection fraction; $\mathrm{HFrEF}$ : heart failure with reduced ejection fraction

presentation observed among Asians during hospital admission, $\mathrm{HF}$ appears to be an emerging threat to Asia if a strategy is not developed to confront and overcome this problem. Although a recent clinical trial has shown the potential mortality benefit of new drugs that act on angiotensin receptor and neprilysin inhibition (e.g. sacubitril/valsartan), ${ }^{(28)}$ data relating to the costeffectiveness of these drugs in Asian populations is still lacking. ${ }^{(29}$

In our study, $38.5 \%$ of patients were in the HFrEF group. The lower proportion of patients in this group may be related to the relatively high number of patients with comorbid diseases such as diabetes mellitus and high blood pressure. This finding is similar to that of the INTER-CHF (International Congestive Heart Failure) study, which showed that the proportion of HFrEF in Southeast Asian populations was 39\%, and that there was a greater proportion of comorbid conditions in Southeast Asian patients. ${ }^{(8)}$ Our study found that patients with HFrEF had a higher mortality rate than those with HFpEF. Data from Olmsted County, Minnesota, in the United States showed that long-term mortality over five years was similar between HFrEF and HRpEF, with more than half of all patients deceased after five years. ${ }^{(30)}$ The higher mortality rate in patients with HFrEF in our study was mainly due to increased cardiovascular mortality, although non-cardiovascular mortality also had a similar trend (Fig. 3). $\mathrm{HFpEF}$ has been reported as a contributing factor in approximately $40 \%-50 \%$ of HF cases. ${ }^{(30,31)}$ A recent study indicated that the prevalence and proportion of HFpEF increased with age, so that there was a greater proportion of HFpEF than HFrEF among the elderly. ${ }^{(32)}$ Long-term mortality rates in patients with HFrEF or HFpEF were found to be similar, although there was a trend towards higher mortality in patients with HFrEF. ${ }^{(31)}$ This is similar to the results from our study; however, the five-year mortality rate in a previous report ${ }^{(31)}$ was higher than our rate.

Our study has some limitations. First, it had a relatively small sample size, obtained by tracking the data of patients enrolled in the Thai ADHERE in 2006. However, that baseline data was selected for this study because we consider the quality of Thai ADHERE data to be high. Moreover, the 2006 data was collected from 18 medical centres located across Thailand and can, therefore, be regarded as representative of $\mathrm{HF}$ care and outcomes in Thailand in 2006. Second, as mortality data was collected from the Thailand Bureau of Registration Administration, the quality of the data on the death certificate depended on the judgement and skill of the physician who was in charge at the time of death.

In conclusion, the ten-year mortality rate in Thai patients admitted for acute decompensated HF was $73.3 \%$. Many factors were found to be independently associated with increased mortality.

\section{ACKNOWLEDGEMENTS}

The Thai ADHERE was endorsed by the Heart Association of Thailand under the Royal Patronage of H.M. the King and the Heart Failure Council of Thailand. The Thai ADHERE is financially supported by the Clinical Research Collaboration Network of Thailand, Janssen-Cilag Limited (Thailand), and the Heart Association of Thailand under the Royal Patronage of H.M. the King.

\section{REFERENCES}

1. Oliva F, Mortara A, Cacciatore G, et al; IN-HF Outcome Investigators. Acute heart failure patient profiles, management and in-hospital outcome: results of the Italian Registry on Heart Failure Outcome. Eur J Heart Fail 2012; 14:1208-17.

2. Ponikowski P, Voors AA, Anker SD, et al; Authors/Task Force Members; Document Reviewers. 2016 ESC guidelines for the diagnosis and treatment of acute and chronic heart failure: the task force for the diagnosis and treatment of acute and chronic heart failure of the European Society of Cardiology (ESC). Developed with the special contribution of the Heart Failure Association (HFA) of the ESC. Eur J Heart Fail 2016; 18:891-975.

3. Ponikowski $\mathrm{P}$, Anker SD, AlHabib KF, et al. Heart failure: preventing disease and death worldwide. ESC Heart Fail 2014; 1:4-25.

4. Heidenreich PA, Albert NM, Allen LA, et al; American Heart Association Advocacy Coordinating Committee; Council on Arteriosclerosis, Thrombosis and Vascular Biology; Council on Cardiovascular Radiology and Intervention; Council on Clinical Cardiology; Council on Epidemiology and Prevention; Stroke Council. Forecasting the impact of heart failure in the United States: a policy statement from the American Heart Association. Circ Heart Fail 2013; 6:606-19.

5. Ziaeian B, Fonarow GC. Epidemiology and aetiology of heart failure. Nat Rev Cardiol 2016; 13:368-78.

6. Rajadurai J, Tse HF, Wang $\mathrm{CH}$, et al. Understanding the epidemiology of heart failure to improve management practices: an Asia-Pacific perspective. J Card Fail 2017; 23:327-39. 
7. Laothavorn P, Hengrussamee K, Kanjanavanit R, et al; Thai ADHERE Registry working groups. Thai Acute Decompensated Heart Failure Registry (Tha ADHERE). CVD Prev Control 2010; 5:89-95.

8. Dokainish H, Teo K, Zhu J, et al; INTER-CHF Investigators. Global mortality variations in patients with heart failure: results from the International Congestive Heart Failure (INTER-CHF) prospective cohort study. Lancet Glob Health 2017; 5:e665-72.

9. Tromp J, Tay WT, Ouwerkerk W, et al; ASIAN-HF authors. Multimorbidity in patients with heart failure from 11 Asian regions: a prospective cohort study using the ASIAN-HF registry. PLoS Med 2018; 15:e1002541.

10. Moleerergpoom W, Hengrussamee K, Piyayotai D, et al. Predictors of in-hospital mortality in acute decompensated heart failure (Thai ADHERE). J Med Assoc Thai 2013; 96:157-64.

11. Atherton JJ, Hayward CS, Wan Ahmad WA, et al; ADHERE International-Asia Pacific Scientific Advisory Committee. Patient characteristics from a regiona multicenter database of acute decompensated heart failure in Asia Pacific (ADHERE International-Asia Pacific). J Card Fail 2012; 18:82-8.

12. Adams KF Jr, Fonarow GC, Emerman CL, et al; ADHERE Scientific Advisory Committee and Investigators. Characteristics and outcomes of patients hospitalized for heart failure in the United States: rationale, design, and preliminary observations from the first 100,000 cases in the Acute Decompensated Heart Failure National Registry (ADHERE). Am Heart J 2005 149:209-16.

13. Nieminen MS, Brutsaert D, Dickstein K, et al; EuroHeart Survey Investigators; Heart Failure Association, European Society of Cardiology. EuroHeart Failure Survey II (EHFS II): a survey on hospitalized acute heart failure patients: description of population. Eur Heart J 2006; 27:2725-36.

14. Yancy CW, Jessup M, Bozkurt B, et al. 2017 ACC/AHA/HFSA focused update of the 2013 ACCF/AHA guideline for the management of heart failure: a report of the American College of Cardiology/American Heart Association Task Force on Clinical Practice Guidelines and the Heart Failure Society of America. J Am Coll Cardiol 2017; 70:776-803.

15. Lam CS, Teng TK, Tay WT, et al. Regional and ethnic differences among patients with heart failure in Asia: the Asian sudden cardiac death in heart failure registry. Eur Heart J 2016; 37:3141-53.

16. Roger VL, Weston SA, Redfield MM, et al. Trends in heart failure incidence and survival in a community-based population. JAMA 2004; 292:344-50.

17. Sato N. Epidemiology of heart failure in Asia. Heart Fail Clin 2015; 11:573-9.

18. Loehr LR, Rosamond WD, Chang PP, Folsom AR, Chambless LE. Heart failure incidence and survival (from the Atherosclerosis Risk in Communities study). Am J Cardiol 2008; 101:1016-22.
19. Robertson J, McElduff P, Pearson SA, et al. The health services burden of heart failure: an analysis using linked population health data-sets. BMC Health Serv Res 2012; 12:103.

20. Butler J, Kalogeropoulos A. Worsening heart failure hospitalization epidemic we do not know how to prevent and we do not know how to treat! J Am Coll Cardiol 2008; 52:435-7.

21. Joffe SW, Webster K, McManus DD, et al. Improved survival after heart failure: a community-based perspective. J Am Heart Assoc 2013; 2:e000053.

22. Jhund PS, Macintyre K, Simpson CR, et al. Long-term trends in first hospitalization for heart failure and subsequent survival between 1986 and 2003: a population study of 5.1 million people. Circulation 2009; 119:515-23.

23. MacIntyre K, Capewell S, Stewart S, et al. Evidence of improving prognosis in heart failure: trends in case fatality in 66,547 patients hospitalized between 1986 and 1995. Circulation 2000; 102:1126-31.

24. Guo Y, Lip GY, Banerjee A. Heart failure in East Asia. Curr Cardiol Rev 2013; 9:112-22.

25. Maggioni AP, Dahlström U, Filippatos G, et al; Heart Failure Association of the European Society of Cardiology (HFA). EURObservational Research Programme: regional differences and 1-year follow-up results of the Heart Failure Pilot Survey (ESC-HF Pilot). Eur J Heart Fail 2013; 15:808-17.

26. Komajda M, Cowie MR, Tavazzi L, et al; QUALIFY Investigators. Physicians' guideline adherence is associated with better prognosis in outpatients with heart failure with reduced ejection fraction: the QUALIFY international registry. Eur J Heart Fail 2017; 19:1414-23.

27. Ouwerkerk W, Voors AA, Anker SD, et al. Determinants and clinical outcome of uptitration of ACE-inhibitors and beta-blockers in patients with heart failure: a prospective European study. Eur Heart J 2017; 38:1883-90.

28. McMurray JJ, Packer M, Desai AS, et al; PARADIGM-HF Investigators and Committees. Angiotensin-neprilysin inhibition versus enalapril in heart failure. N Engl J Med 2014; 371:993-1004.

29. King JB, Shah RU, Bress AP, Nelson RE, Bellows BK. Cost-effectiveness of sacubitril-valsartan combination therapy compared with enalapril for the treatment of heart failure with reduced ejection fraction. JACC Heart Fail 2016; 4:392-402.

30. Owan TE, Hodge DO, Herges RM, et al. Trends in prevalence and outcome of heart failure with preserved ejection fraction. N Engl J Med 2006; 355:251-9.

31. Fonarow GC, Stough WG, Abraham WT, et al; OPTIMIZE-HF Investigators and Hospitals. Characteristics, treatments, and outcomes of patients with preserved systolic function hospitalized for heart failure: a report from the OPTIMIZE-HF Registry. J Am Coll Cardiol 2007; 50:768-77.

32. Oktay AA, Rich JD, Shah SJ. The emerging epidemic of heart failure with preserved ejection fraction. Curr Heart Fail Rep 2013; 10:401-10. 